$\Rightarrow$ MEMBRANOUS NEPHROPATHY

\section{Genetics of MN}

Membranous nephropathy (MN) is an autoimmune kidney disease that has an underlying genetic component; however, the only genome-wide association study (GWAS) to date was of limited size and included only individuals of European ancestry. Now, a large, multinational GWAS has provided greater insights into the genetic architecture of MN. "Our GWAS identified novel risk loci for MN, refined ethnicityspecific effects at the HLA locus and supports a regulatory function of the PLA2R1 risk haplotype," says Krzysztof Kiryluk. "Moreover, we were able to generate a genetic risk score (GRS) that had excellent diagnostic properties when combined with an anti-PLA2R serum test."

Kiryluk explains that their study required a large-scale international collaboration to assemble biopsy-diagnosed cohorts of sufficient size. "This study included 3,782 cases and 9,038 controls recruited across East Asia, Europe and North America." The researchers identified two novel risk loci: NFKB1 and IRF4. "These genes participate in a common immune regulatory pathway; our findings clearly establish a role of this pathway in MN pathogenesis," says Kiryluk. They also identified ethnicityspecific effects at the HLA locus, defining $D R B 1^{\star} 1501$ as a major risk allele in East Asians, DQA $1{ }^{\star} 0501$ in Europeans and DRB1*0301 in both ethnicities, which the researchers propose could suggest the presence of different immunological triggers in different populations. Fine mapping of the PLA2R1 locus showed that the risk variant intersects a predicted regulatory element. Although this variant was associated with suppressed antigen expression across multiple tissues, it seems to increase expression of PLA2R1 in the kidney.

Development of a GRS showed that the identified variants were highly predictive of disease status, explaining $29 \%$ of disease risk across the cohorts. Finally, the researchers show that combining the GRS with an antiPLA2R ELISA enabled re-classification of $20-37 \%$ of cases in which the ELISA test was either negative or inconclusive. "The complementary information provided by the GRS could potentially spare the need for a kidney biopsy in this large subgroup of patients," explains Kiryluk. "However, future efforts to test this strategy in more diverse populations will be important."

Susan J. Allison

ORIGINAL ARTICLE Xie, J. et al. The genetic architecture of membranous nephropathy and its potential to improve non-invasive diagnosis. Nat. Commun. 11, 1600 (2020)

\section{Epigenetic control of inflammatory cells}

Inflammation has been associated with metastatic progression in clear cell renal cell carcinoma (ccRCC). Blocking chemokine expression in cancer cells through the inhibition of chromatin remodelling might attenuate inflammatory processes and reduce metastatic spread, according to a preclinical study by Kohei Miyazono and Shogo Ehata.

The researchers demonstrated that, in an orthotopic xenograft mouse model, the use of highly aggressive inflammatory human ccRCC (iRCC) cells (derived through serial orthotopic inoculation in mice) increased the infiltration of primary tumours and lungs by Ly6G ${ }^{+}$neutrophils, compared with the parental RCC (paRCC) cells. An anti-Ly6G antibody not only reduced the number of neutrophils in this model but also decreased the metastatic burden; primary tumour size was not affected.

In vitro, neutrophil migration and survival were enhanced in the presence of supernatants from iRCC cells but not those from paRCC cells, suggesting that iRCC cells might contribute to inflammation by releasing soluble mediators that modulate neutrophil phenotypes. Epigenetic analyses revealed that genes encoding proteins associated with responses to hypoxia, such as hypoxia-inducible factor (HIF), and inflammation, such as nuclear factor- $\kappa \mathrm{B}$ (NF- $\mathrm{B}$ ) and CCAAT/enhancer-binding protein $(\mathrm{C} / \mathrm{EBP})$, were transcriptionally active in iRCC cells. Compared with paRCC cells, expression of CXC chemokines was also increased in iRCC cells, and chromatin precipitation analysis suggested that CXCL8 expression was controlled by a super-enhancer (SE) in these cells.

"Surprisingly, a bromodomain and extra-terminal (BET) inhibitor, which blocks SE-driven transcription, broadly reduced the expression of several chemokines," remarks Ehata. "In vivo, this inhibitor decreased both neutrophil infiltration and lung metastasis."

"BET inhibitors or related compounds might therapeutically target inflammationdriven metastatic spread in patients with advanced RCC," adds Ehata.

Monica Wang

COVID-19

\title{
SARS-CoV-2 infection of kidney organoids prevented with soluble human ACE2
}

The current COVID-19 pandemic is caused by severe acute respiratory syndrome coronavirus 2 (SARS-CoV-2). This virus gains entry to host cells via ACE2, which is expressed by type 2 alveolar epithelial cells in the lungs and by cells in other tissues, including the heart, kidney and blood vessels. Researchers now show that SARS-CoV-2 can infect human blood vessel and kidney organoids and that this infection can be inhibited by human recombinant soluble ACE2 (hrsACE2).

To assess whether hrsACE2 - a decoy protein that has already been tested in phase I and II clinical trials - can inhibit the interaction between SARS-CoV-2 and ACE2, Josef Penninger, Ali Mirazimi, Nuria Montserrat and colleagues administered the recombinant protein to SARS-CoV-2-infected Vero E6 cells. Treatment of cells with hrsACE2 inhibited SARS-CoV-2 infection in a dose-dependent manner and attenuated propagation of the virus.

Based on the hypothesis that the presence of ACE2 in extrapulmonary tissues might explain the multi-organ dysfunction caused by
COVID-19, the researchers developed human capillary organoids from induced pluripotent stem cells, and kidney organoids from human embryonic stem cells. "Importantly, single-cell RNA sequencing of the kidney organoids showed that ACE2 is expressed in different cell populations, including tubular-like cells and podocyte-like cells," says Montserrat. Following infection of the organoids with SARS-CoV-2 the researchers could detect viral RNA that increased in the days following infection, indicative of viral replication. Addition of hrsACE2, however, again reduced SARS-CoV-2 levels in a dose-dependent manner. "Our findings not only suggest that tissue organoids can be used to study the effects of SARS-CoV-2 on extrapulmonary tissues, but also suggest that rhsACE2 might block the virus from entering target cells," says Penninger.

Susan J. Allison

ORIGINAL ARTICLE Monteil, V. et al. Inhibition of SARS-CoV-2 infections in engineered human tissues using clinical-grade soluble human ACE2. Cell https://doi.org/10.1016/j.cell. 2020.04.004 (2020), pre-proof available at https://www.cell.com/ pb-assets/products/coronavirus/CELL_CELL-D-20-00739.pdf 